Check for updates

Cite this: Nanoscale Adv., 2019, 1, 3139

\title{
Iron oxide-based nanostructured ceramics with tailored magnetic and mechanical properties: development of mechanically robust, bulk superparamagnetic materials
}

\author{
Diletta Giuntini, (D) *a Elisa Torresani, ${ }^{\mathrm{b}}$ Kyle T. Chan, ${ }^{\mathrm{c}}$ Malte Blankenburg, ${ }^{\mathrm{d}}$ \\ Lucien Saviot, (D) ${ }^{\mathrm{e}}$ Büsra Bor, ${ }^{a}$ Berta Domènech, (D) ${ }^{a}$ Meir Shachar, ${ }^{c}$ Martin Müller, ${ }^{d}$

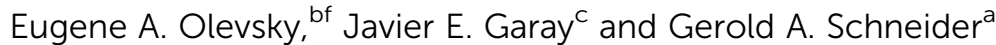

\begin{abstract}
Nanostructured iron-oxide based materials with tailored mechanical and magnetic behavior are produced in bulk form. By applying ultra-fast heating routines via spark plasma sintering (SPS) to supercrystalline pellets, materials with an enhanced combination of elastic modulus, hardness and saturation magnetization are achieved. Supercrystallinity - namely the arrangement of the constituent nanoparticles into periodic structures - is achieved through self-assembly of the organically-functionalized iron oxide nanoparticles. The optimization of the following SPS regime allows the control of organics' removal, necking, iron oxide phase transformations and nano-grain size retention, and thus the fine-tuning of both mechanical properties and magnetic response, up until the production of bulk mm-size superparamagnetic materials.
\end{abstract}

Received 8th April 2019
Accepted 2nd July 2019
DOI: 10.1039/c9na00222g
rsc.li/nanoscale-advances

\section{Introduction}

Nanostructured ceramics are attracting growing attention in materials science and engineering, thanks to their wide spectrum of unique properties and applications. Supercrystalline ceramic-based materials are a sub-category of nanostructured ceramics. They are typically nanocomposites in which ceramic nanoparticles, surface-functionalized with organic ligands, are arranged into periodic structures. Thanks to the nanoscale size of their building blocks and their long-range order architecture, they feature very promising combinations of optical, electronic, magnetic and mechanical properties. ${ }^{1-5}$

Iron oxide is often the material of choice for the inorganic phase, thanks to well-established synthesis routines that allow the production of organically-stabilized iron oxide nanoparticles with narrow monomodal size distribution..$^{6-9}$ As shown later in the present work as well, this is a fundamental

anstitute of Advanced Ceramics, Hamburg University of Technology (TUHH), Denickestr. 15, D-21073 Hamburg, Germany. E-mail: diletta.giuntini@tuhh.de

${ }^{b}$ Department of Mechanical Engineering, San Diego State University (SDSU), 5500 Campanile Dr., San Diego, CA 92182, USA

${ }^{c}$ Department of Mechanical and Aerospace Engineering, University of California, San Diego (UCSD), 9500 Gilman Dr., La Jolla, CA 92093, USA

${ }^{d}$ Institute of Materials Research, Helmholtz-Zentrum Geesthacht, Max-Planck-Str. 1, D21502 Geesthacht, Germany

${ }^{e}$ Laboratoire Interdisciplinaire Carnot de Bourgogne, UMR 6303 CNRS-Université Bourgogne Franche-Comté, 9 Av. A. Savary, BP 47 870, Dijon Cedex F-21078, France ${ }^{f}$ Department of Nanoengineering, University of California, San Diego (UCSD), 9500 Gilman Dr., La Jolla, CA 92093, USA requirement to obtain supercrystalline nanoarchitectures. Iron oxide-based super-crystalline materials have been produced starting from a variety of nanoparticles shapes and iron-phase content, with a prevalence of magnetite $\left(\mathrm{Fe}_{3} \mathrm{O}_{4}\right)$ and maghemite $\left(\gamma-\mathrm{Fe}_{2} \mathrm{O}_{3}\right)$. The resulting supercrystals are typically $2 \mathrm{D}$ or $3 \mathrm{D}$ with sizes in the $10-100 \mu \mathrm{m}$ range, and they find applications in several functional materials. ${ }^{10-20}$ Larger bulk iron oxidebased supercrystalline nanocomposites have also started to be developed for structural purposes, by exploiting the option of crosslinking the organic ligands anchored to the nanoparticles. ${ }^{21}$

Iron oxide-based nanostructured ceramics, typically not supercrystalline, are also being investigated for their magnetic properties. Iron oxide is earth-abundant and can be produced in a variety of phases with distinct magnetic behaviors, ${ }^{22-24}$ allowing room for the production of bulk nanostructured materials with tailored magnetic response. This can be achieved by combining different iron oxide phases or a specific iron oxide phase with an additional matrix material, enabling size control of the magnetic domains, the combination of soft and hard magnetic responses, and optimized exchange coupling. Maintaining the nanostructure in bulk components is also potentially promising to preserve superparamagnetism in threedimensional macroscale materials, paving the way for the next generation of magnetic cores for transformers in energy applications. ${ }^{25-32}$

It is important to point out that such outcomes have typically been achieved thanks to the SPS/CAPAD technology (spark plasma sintering/current-activated pressure-assisted densification), 
a powder consolidation technique that relies on an electric current to achieve sintering. Depending on the material being processed, the electric current either only provides heating via Joule effect when crossing the conductive tooling that surrounds the powder samples, or activates additional densification-enhancing mechanisms. In any of such cases, SPS/CAPAD is characterized by high heating rates and overall greatly reduced processing times, enabling the development of novel materials with nanoscale grains, metastable phases, fine-tuned structures and constituents' combinations. $^{33-43}$

In the present work, SPS was applied to supercrystalline iron oxide/oleic acid nanocomposites, in order to obtain nanostructured iron oxide-based ceramics with fine-tuned mechanical and magnetic properties. The predominant iron oxide phase was magnetite $\left(\mathrm{Fe}_{3} \mathrm{O}_{4}\right)$. SPS guaranteed the preservation of the supercrystalline arrangement up to temperatures much higher than those allowed by conventional processing. The combination of such ultra-fast processing routines and the narrow particle size distribution led to hampered grain growth, and thus bulk nano-grained materials with enhanced mechanical properties. This combined with a broad range of magnetic responses, obtained by opportunely varying the SPS process parameters, ultimately leading to bulk ceramics with controlled grain size and tailored mechanical and magnetic behavior. The excellent nanostructural control is exemplified by the preservation of the superparamagnetic response typical of the constituent nanoparticles in the bulk nanocomposites, an extremely rare result with potential applications in electrically insulating high magnetic response substrates., ${ }^{\mathbf{9 4 4 - 5 1}}$

\section{Materials and methods}

The starting material is a suspension of nearly spherical iron oxide (magnetite, $\mathrm{Fe}_{3} \mathrm{O}_{4}$ ) nanoparticles functionalized with oleic acid $\left(\mathrm{C}_{18} \mathrm{H}_{34} \mathrm{O}_{2}\right)$, a relatively short-chained (1-2 $\mathrm{nm}$ ) organic ligand anchored to the nanoparticles' surface through its carboxylic group. The phase content of the iron oxide nanoparticles as magnetite-dominated has been assessed via X-ray photoelectron spectroscopy in a previous work of this same group, and is additionally verified in the course of this study. ${ }^{21}$ Three different suspension batches (produced by CAN GmbH, Germany) were employed, with slightly different particle sizes. The impact of such a parameter on the final material properties will be clarified in the following sections.

In order to form bulk supercrystalline pellets, a sequence of two steps was performed: self-assembly via slow solvent evaporation in an open die/punch assembly, followed by uniaxial pressing of the dried self-assembled material. A pressure of $50 \mathrm{MPa}$ was applied at a temperature of $150^{\circ} \mathrm{C}$, which a previous empirical study has revealed to ensure optimal rheology of the oleic acid during compaction of the pellets. ${ }^{21}$ The oleic acid content after this stage is $\sim 11 \mathrm{wt} \%$. The pellets were subsequently processed via SPS (Dr. Sinter 515 SPS, Fuji Electronic Industrial Co., Ltd., Japan). The holding temperature ranged between 300 and $550{ }^{\circ} \mathrm{C}$, the holding time between 0 and $10 \mathrm{~min}$, the applied pressure was 0 or $45 \mathrm{MPa}$, the heating ramp was $100{ }^{\circ} \mathrm{C} \mathrm{min}^{-1}$, and the cooling stage consisted of machine shutdown to let the sample-tooling assembly slowly reach room temperature. When no pressure is applied, the SPS process is typically termed FPSPS (free pressure-less SPS). Two different graphite tooling setups were used to accommodate the samples during SPS: a $15 \mathrm{~mm}$-diameter die/punches assembly for regular SPS and a $10 \mathrm{~mm}$-diameter die with T-shaped punches for FPSPS. In the first case, the green pellets had a $15 \mathrm{~mm}$ diameter and a height of 4-5 mm, while in the latter 4-5 mm portions of the green pellets were used. Graphite foil was used to prevent adhesion at the interfaces between samples and tooling. The process was conducted in vacuum.

The supercrystalline nanostructure was characterized before and after SPS via scanning electron microscopy (SEM) and synchrotron radiation-based small-angle X-ray scattering (SAXS). Thermogravimetric analysis (TGA) was used to assess the final organic content $\left(25-900{ }^{\circ} \mathrm{C}\right.$ at $1{ }^{\circ} \mathrm{C} \min ^{-1}$ rate in nitrogen gas atmosphere). A combination of Raman spectroscopy and synchrotron radiation-based wide-angle X-ray scattering (WAXS) provided the details of the chemical composition and iron oxide phase content.

Small-angle and wide-angle X-ray scattering experiments were performed at the High Energy Materials Science (HEMS, P07) beamline, operated by Helmholtz-Zentrum Geesthacht at the PETRA III storage ring at the Deutsches ElektronenSynchrotron (DESY). ${ }^{52}$ The energy of the incident beam was $87.1 \mathrm{keV}$ (wavelength: $0.01423 \mathrm{~nm}$ ) and the cross section was 0.2 $\times 0.2 \mathrm{~mm}^{2}$. A two-dimensional PerkinElmer detector with a pixel size of $200 \mu \mathrm{m}$ was placed at sample-to-detector distances of $3400 \mathrm{~mm}$ (SAXS) and $1720 \mathrm{~mm}$ (WAXS), respectively, to detect the scattering signal. Fit $2 \mathrm{~d}^{53}$ was used for the data reduction. For the calculation of the particle sizes and the determination of the supercrystalline lattice parameters the software Scatter ${ }^{54}$ was used, assuming a hard sphere model and a log-normal size distribution of the particle radius. Full pattern Rietveld refinement for the WAXS data was conducted using the software MAUD. ${ }^{55}$

Raman spectroscopy was conducted with a Renishaw inVia setup and a laser power optimized to avoid material oxidation while preserving an adequate signal-to-noise ratio. A $633 \mathrm{~nm}$ laser excitation was thus selected. The laser power density on the samples was $\sim 10 \mathrm{~kW} \mathrm{~cm}{ }^{-2}$. The spectral regions of interest were $<1000 \mathrm{~cm}^{-1}$ for iron oxide and $\sim 1500 \mathrm{~cm}^{-1}$ for the organic material (C-C bonds).

The mechanical properties were characterized in terms of elastic modulus and hardness via nanoindentation with an Agilent Nano Indenter G200 (Agilent, Santa Clara, CA, USA). A Berkovich tip and the continuous stiffness measurement (CSM) mode were used to perform twenty $1000 \mathrm{~nm}$-deep indents in each sample.

The magnetic behavior was assessed by means of a vibratingsample magnetometer (VSM). The consolidated samples were first polished into millimeter-sized cubes, and then mounted in a diamagnetic holder. The samples were demagnetized using an alternating magnetic field with decreasing magnitude. Magnetization-applied field $(M-H)$ loops were collected for each sample with applied fields from -10 to $10 \mathrm{kOe}$. The magnetic moments were normalized by mass. 


\section{Results and discussion}

Fig. 1 shows typical nanostructures of the obtained $\mathrm{Fe}_{3} \mathrm{O}_{4} /$ oleic acid samples, after pressing. In part (A) a single supercrystal is shown, while in part (B) the boundary between two differently oriented supercrystals is depicted. The bulk samples produced in this work are poly-supercrystalline, in which - as inferred via SEM - several supercrystalline domains (or "grains") with sizes in the 10-100 $\mu \mathrm{m}$ order of magnitude coexist.

In order for supercrystallinity to be achieved, it is essential that the nanoparticles size distribution is very narrow. Both the initial suspension batches and the green pellets obtained with the different batches were tested with SAXS before SPS, confirming this statement. Fig. 2A shows the size distribution of the various functionalized nanoparticles that were employed, as determined from the starting suspensions. The size distribution is inferred from SAXS data by analyzing the form factor of the nanoparticles, whose spherical shape is most clearly visible as a slow oscillation at higher $q$-values (e.g. in the red curve of Fig. 2B). The degree of supercrystallinity is reflected in two other features of the SAXS curves in Fig. 2B. The first is the peak at low $q$-values. This peak (present in the red and blue curves and cut off in the green curve due to a different X-ray beam stop position during the experiment) is part of the structure factor caused by interference of the scattering of different nanoparticles. The sharper this peak (see Batch 3), the more clearly the main distance between neighboring nanoparticles is defined, even though a randomly organized assembly of nanoparticles will also show such a peak. The second feature consists of the additional sharp reflections at higher $q$-values, which appear in presence of a well-ordered supercrystalline arrangement. These are found for Batch 1 and 3. Their smearing, in Fig. 2B and in the following figures, implies loss of long-range order in the superlattice. One can therefore conclude that these nanoparticles batches with narrow size distribution yield supercrystalline pellets, while samples obtained with Batch 2 are mostly amorphous, with localized supercrystalline domains, as inferred via SEM. This can be attributed to the greater amount of imperfections in the arrangement of nanoparticles with varying diameters. Such a qualitative initial level of supercrystallinity will need to be kept in mind when evaluating the effects of SPS on the material's nanostructural arrangement.

SAXS also provided more detailed quantitative information about the supercrystalline structure. The obtained superlattice was consistently of the face-centered cubic (FCC) type. The nanoparticles radius was $8.9 \pm 0.8 \mathrm{~nm}, 9.1 \pm 1.4$ and $7.8 \pm$ $0.9 \mathrm{~nm}$ for Batches 1, 2 and 3, respectively, while the organic interface between the ceramic nanoparticles (difference between nearest neighbor distance and particle diameter) was $\sim 1 \mathrm{~nm}$ in all cases.

Post-SPS SAXS revealed the important role played by temperature, holding time and pressure towards the preservation of the supercrystalline lattice. Fig. 3 summarizes these effects. As a reference, part (A) shows the effect of heat treatment in a conventional furnace $\left(1^{\circ} \mathrm{C} \mathrm{min}{ }^{-1}\right.$ heating and cooling ramps, $18 \mathrm{~min}$ hold at the listed temperatures, in nitrogen atmosphere), while part (B) shows the evolution of the material's nanostructure when FPSPS is applied with increasing temperatures and varying holding times. Note first of all that, for both conventional heat treatment and FPSPS, these plots report results of one single pellet, which was divided into multiple portions and processed at the conditions reported in the figure. For conventional heat treatment, the pellet had been produced with Batch 1, while for the FPSPSed pellet Batch 2 had been used. This is clearly reflected in the pre-SPS supercrystallinity conditions, as given already in Fig. 2, which show superlattice reflection peaks in Fig. 3A, and not in 3B. Again, the different $q$ vector ranges are due the different beam stop positions during SAXS tests. The important point here lies nevertheless in the comparison between scattering signals within each plot. While the long heating times typical of conventional processing lead to a significant loss of supercrystallinity already at $350{ }^{\circ} \mathrm{C}$, with FPSPS the nanoparticles' size, shape and arrangement are better preserved up to $450{ }^{\circ} \mathrm{C}$. This appears to be a critical temperature, as shifting from 1 minute of holding time to 5 and 10 minutes causes peak smearing. In all these cases, peak smearing is interpreted as sintering of the iron oxide nanoparticles. Note that even if magnetite has a melting point of $1595{ }^{\circ} \mathrm{C}$, the nano-size of the particles makes them extremely prone to sintering already at such low temperatures.
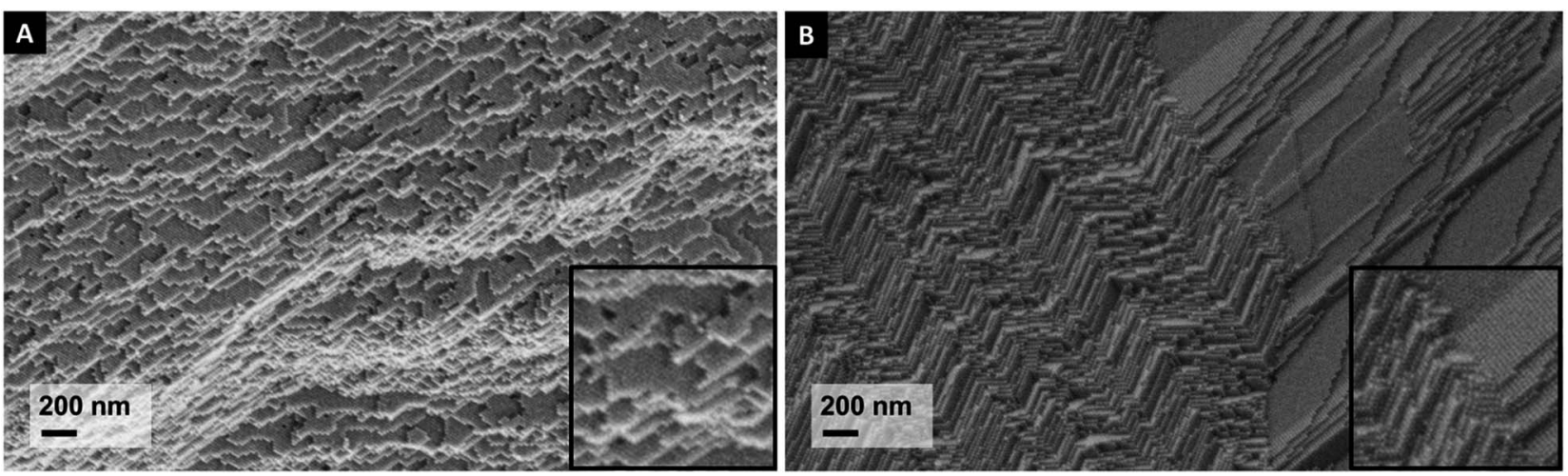

Fig. 1 Nanostructure of the pre-SPS Fe $\mathrm{O}_{4}$ /oleic acid material: (A) a single supercrystalline domain; (B) two supercrystalline domains interfacing. 

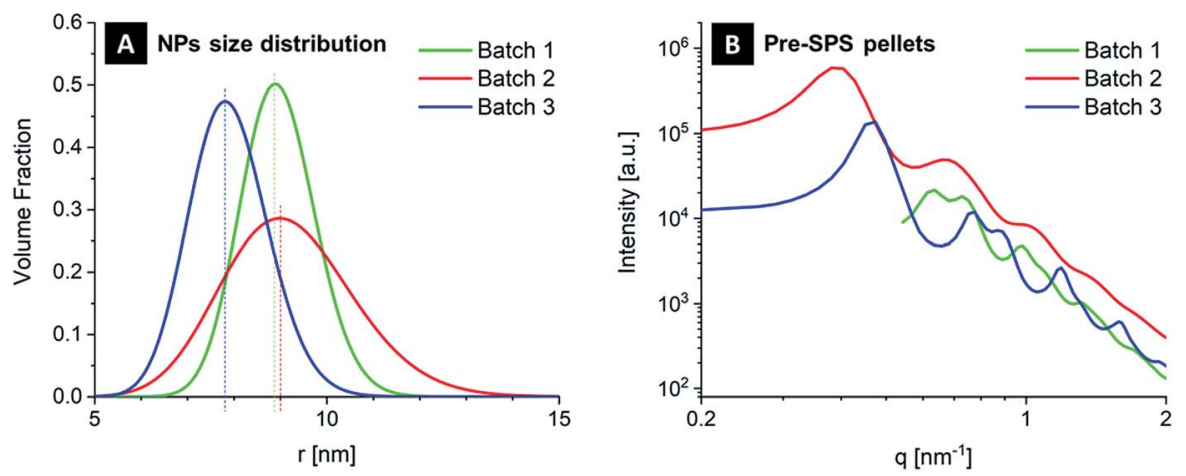

Fig. 2 SAXS analysis of (A) the functionalized magnetite nanoparticles suspensions (three batches) and (B) the supercrystallinity of the resulting bulk materials. In Batches 1 and 3 of part (B) sharper peaks correspond to materials with a high degree of supercrystallinity.

Indeed, while conventional sintering of $\mu \mathrm{m}$-sized powders typically requires temperatures around $0.7 T_{\mathrm{m}, \mathrm{b}}(70 \%$ of the melting temperature of the bulk material), the well-known decrease in the melting point of nanoparticles $\left(T_{\mathrm{m}, \mathrm{p}}\right)$ with respect to a bulk - expressed as $T_{\mathrm{m}, \mathrm{p}}=T_{\mathrm{m}, \mathrm{b}}-\alpha / d$, where $d$ is the particle diameter and $\alpha$ a material constant - combined with the densification-enhancing mechanisms characteristic of SPS, ${ }^{37}$ has shown that densification is already possible at temperatures in the $0.24-0.4 T_{\mathrm{m}, \mathrm{b}}$ range..$^{56}$ Sintering can start as soon as the oleic acid preventing direct contact between the ceramic nanoparticles is removed. According to TGA data, most of the organic material is eliminated in the $150-400{ }^{\circ} \mathrm{C}$ temperature range, with the maximum loss starting at $300{ }^{\circ} \mathrm{C} .{ }^{21}$ Such a wide temperature window for the removal of oleic acid is usually associated with the presence of ligands that are differently bound to the nanoparticles' surface, ${ }^{57}$ to the ligands' decomposition or desorption at the surface, ${ }^{58}$ and to their high confinement in-between the nanoparticles themselves. Another important factor to keep in mind for the sintering behavior of the nanocomposites is the narrow and monomodal size distribution of the nanoparticles, which hampers grain growth after the onset of densification..$^{59}$

The application of pressure at high temperatures has a more immediate effect on superlattice disruption, as Fig. 3C shows for the case of $350{ }^{\circ} \mathrm{C}$ as holding temperature. A pressure of
$45 \mathrm{MPa}$ is sufficient to remove the supercrystalline structure almost completely. This is due to the accelerated consolidation of the ceramic phase, also favored by a pressure-induced rearrangement of the ceramic particles within the fluid oleic acid (the boiling point of oleic acid is $360{ }^{\circ} \mathrm{C}$, even though its rheology and physical properties might be altered here due to the anchoring to the iron oxide nanoparticles and the confinement in a nm-size area). Reduced holding times and higher heating rates can be used to maintain supercrystallinity also when a pressure is applied.

For both FPSPS at the highest processing temperature (550 ${ }^{\circ} \mathrm{C}$ ) and pressure-assisted (conventional) SPS up to $350{ }^{\circ} \mathrm{C}$, the supercrystallinity loss is accompanied by the development of an interesting microstructure, in which the grain size is still in the nanoscale and texturing reminiscent of the supercrystalline "terraces" is observed. Increasing further temperature, pressure and/or holding time leads instead to grain growth. Fig. 4 shows these effects in fracture surfaces of samples processed via FPSPS and pressure-assisted SPS $(45 \mathrm{MPa})$ at $350{ }^{\circ} \mathrm{C}$ and $550{ }^{\circ} \mathrm{C}$. The reported grain sizes were obtained via WAXS, by using the "Delft" model, ${ }^{60}$ which attributes the Lorentzian broadening to the finite crystallite size. Grain growth is clearly inhibited up to $550{ }^{\circ} \mathrm{C}$ for FPSPS and $350{ }^{\circ} \mathrm{C}$ for pressure-assisted SPS. Supercrystallinity is still present for FPSPS- $3500^{\circ} \mathrm{C}$ samples (A), quasinegligible grain growth and nano-structure texturing can be
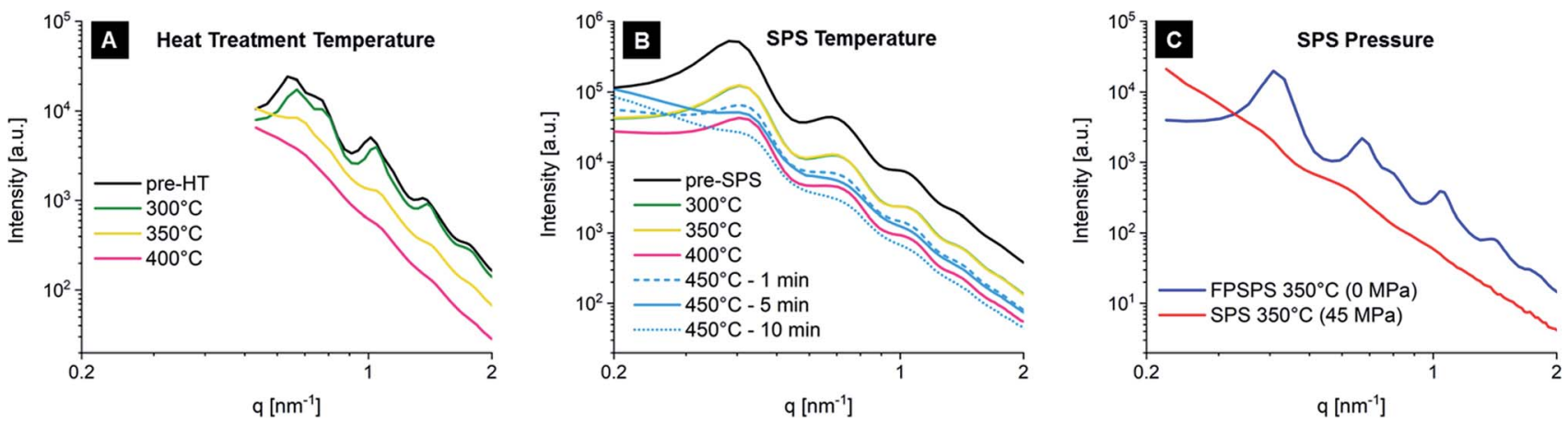

Fig. 3 SAXS analysis of the effect on supercrystallinity of (A) temperature during conventional heat treatment (Batch 1); (B) temperature during SPS (Batch 2); (C) pressure during SPS (Batch 1). 


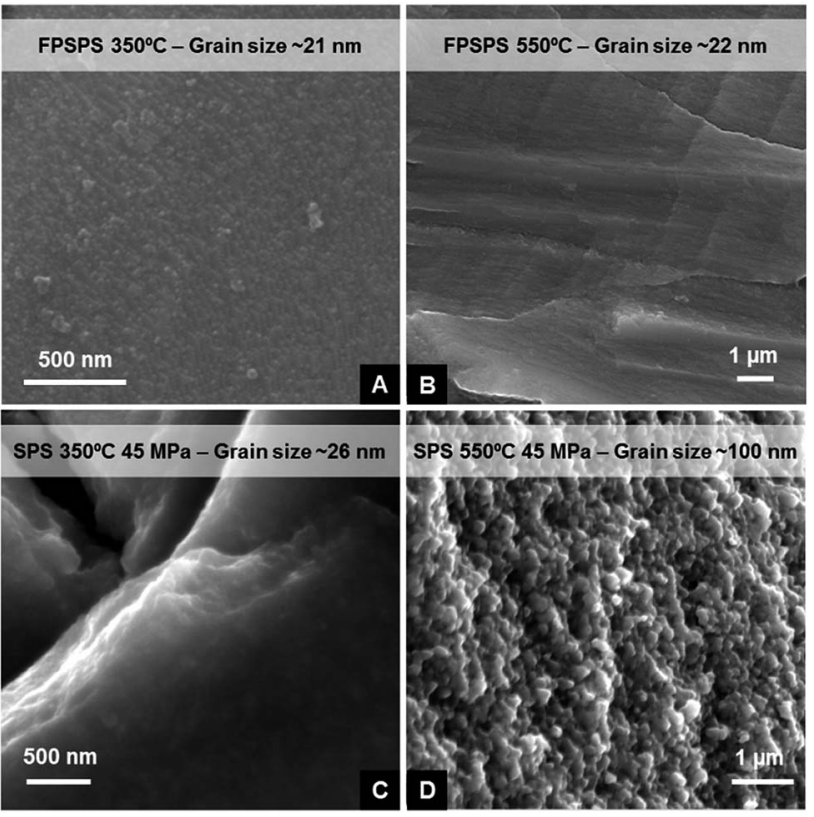

Fig. 4 Microstructure of samples processed via (A) FPSPS at $350{ }^{\circ} \mathrm{C}$, (B) FPSPS at $550{ }^{\circ} \mathrm{C}$, (C) SPS with $45 \mathrm{MPa}$ at $350^{\circ} \mathrm{C}$, and (D) SPS with $45 \mathrm{MPa}$ at $550^{\circ} \mathrm{C}$.

observed in parts (B) and (C) of the figure, while pressureassisted SPS at $550{ }^{\circ} \mathrm{C}$ leads to sintering and grain growth, even if the final grain size is still extremely fine, in the sub- $\mu \mathrm{m}$ range.

Fig. 5 shows the evolution of density and oleic acid content with SPS temperature, as measured via Archimedes method and TGA, respectively. The plotted data points are an average including FPSPS and SPS samples. The expected decrease in oleic acid content is observed, reduced almost to zero at $550{ }^{\circ} \mathrm{C}$. The removal of oleic acid from the samples could also be detected during the SPS process, in the form of a slight increase of the SPS chamber pressure. Being the process carried out in vacuum, the pump allowed quick removal of the evaporated organic ligands. Relative density is the ratio between the density measured via Archimedes method and the theoretical density. It increases with SPS temperature, with the exception of $300{ }^{\circ} \mathrm{C}$.
The drop recorded at this temperature can be explained with a removal of oleic acid not accompanied by consolidation. The nanoparticles then approach each other and start sintering at the higher SPS temperatures. Note that the relative densities $(\rho)$ were all calculated with respect to theoretical densities $\left(\rho_{\text {th }}\right)$ that considered the effective content of organic material as recorded by TGA, as $\rho_{\text {th }}=\phi_{\mathrm{OA}} \rho_{\mathrm{OA}}+\left(1-\phi_{\mathrm{OA}}\right) \rho_{\mathrm{Fe}_{3} \mathrm{O}_{4}}$, where $\phi_{\mathrm{OA}}$ is the volume fraction of oleic acid. Part (A) of Fig. 5 shows how theoretical density evolves with oleic acid content. The $55 \mathrm{vol} \%$ of iron oxide for pre-SPS conditions is in good agreement with SAXS results. The densities of the two material constituents (at $25^{\circ} \mathrm{C}$ ) are $5.18 \mathrm{~g} \mathrm{~cm}^{-3}$ for magnetite and $0.89 \mathrm{~g} \mathrm{~cm}^{-3}$ for oleic acid. Even if the data reported in the figure are averaged for FPSPS and pressure-assisted SPS, it is worth mentioning that the application of a pressure during processing led to higher densities and higher residual content of oleic acid (with the exception of $550{ }^{\circ} \mathrm{C}$ holding temperature, at which no organic phase is left even when pressure is not applied). This is likely due to the two different tooling setups used for FPSPS and SPS.

All the above considerations on densification, organic content and grain size are reflected in the materials' mechanical properties. Elastic modulus and hardness, as measured via nanoindentation, are given in Fig. 6 for both FPSPS (in blue in the figure) and SPS samples (in red), together with pre-SPS material (black) and the values for samples processed with conventional heat treatment as references (light blue band). ${ }^{21}$ Several interesting features can be observed. In general, the onset of densification of the ceramic phase leads to the expected increase in elastic modulus and hardness, until grain growth becomes significant $\left(550^{\circ} \mathrm{C}\right)$. However, samples processed with SPS at $300{ }^{\circ} \mathrm{C}$, both with and without pressure, show lower stiffness and hardness with respect to their conventionally treated counterparts, probably due to enhanced organic removal, annihilating the effect of the organic ligands' crosslinking, and not compensated for by densification, as mentioned above. Ligand crosslinking is indeed responsible for the overall material's strengthening when moderate heating is applied, by creating a C-C bond between neighboring oleic acid chains and thus limiting the nanoparticles mobility. ${ }^{21}$ This then suggests that for $350{ }^{\circ} \mathrm{C}$, the obtained mechanical properties very high for ceramic/organic nanocomposites - which are
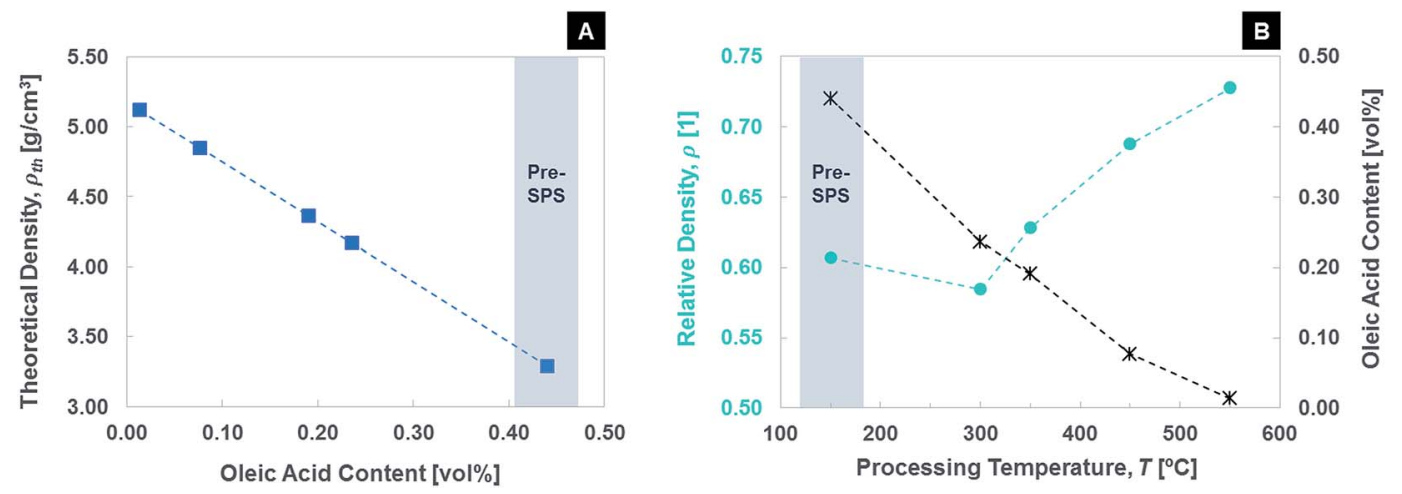

Fig. 5 (A) Theoretical density as function of oleic acid content (varying with the applied processing temperature); (B) relative density and oleic acid content as function of the processing temperature. 

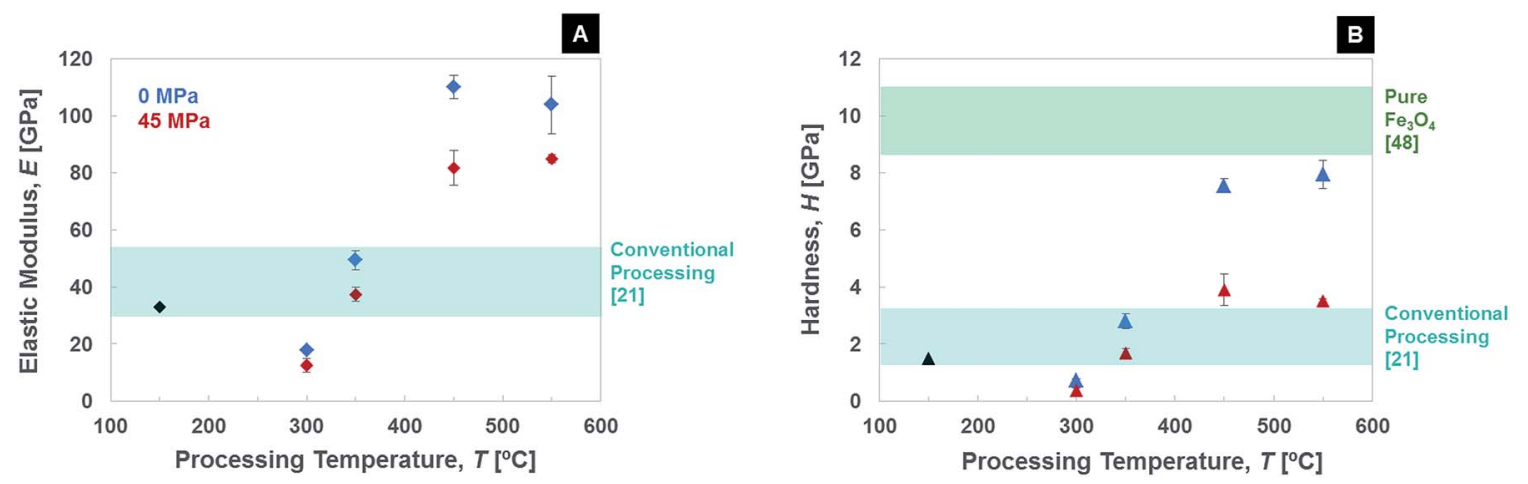

Fig. 6 Elastic modulus (A) and hardness (B) of the samples processed with conventional techniques (black), FPSPS (blue) and pressure-assisted SPS (red).

comparable to the ones obtained with conventional heat treatment, are not due to organic crosslinking but onset of necking between the magnetite nanoparticles. Such a conclusion underlines the fundamental difference between slow heat treatment $\left(1{ }^{\circ} \mathrm{C} \min ^{-1}\right)$ and SPS $\left(100{ }^{\circ} \mathrm{C} \min ^{-1}\right)$, since the application of the same maximum temperature has very distinct effects in the two cases. An interesting result is the FPSPS- $450{ }^{\circ} \mathrm{C}$ one, for which elastic modulus of $110 \pm 4 \mathrm{GPa}$ and hardness of $7.55 \pm 0.25 \mathrm{GPa}$ are measured, while SAXS had detected preservation of the nanoparticles' arrangement, shape and size distribution. Such a hardness value approaches what reported in the literature for pure magnetite, ${ }^{61}$ despite the still high porosity present in such a material, most likely thanks to the grain size in the nanometric scale. This comparison takes into account the well-known indentation size effect. ${ }^{62}$ The combination of densification triggering and nano-grain sizes with organic material removal is thus responsible for a sharp increase in the material's mechanical performance. Interestingly, when pressure is applied, a drop in Young's modulus and hardness is observed, in line with the fact that a $2.6 \mathrm{wt} \%$ of oleic acid - the mechanically weaker phase - is still present in the samples. Increasing the SPS temperature up to $550^{\circ} \mathrm{C}$ translates in further densification and grain growth, and thus in properties comparable to the $450{ }^{\circ} \mathrm{C}$ one, but with no trace of supercrystallinity left. Note that the data plotted in Fig. 6 are relative to a 5 min holding time. The mechanical behaviour of the consolidated materials has here been assessed only in terms of elastic modulus and hardness, but supercrystalline and nanostructured materials are showing promising potential for the development of plastic deformation mechanisms. An in-depth analysis of the constitutive behaviour of these supercrystalline nanocomposites is the object of ongoing work.

The magnetic properties of the nanocomposites can also be controlled by adjusting the SPS parameters. Fig. 7 displays the various magnetic behaviors achieved, in terms of hysteresis magnetization-applied field curves, as resulting from VSM measurements. All the obtained materials show high saturation magnetization $\left(M_{\mathrm{s}}\right)$ and low coercivity $\left(H_{\mathrm{c}}\right)$. The $M_{\mathrm{s}}$ ranges from

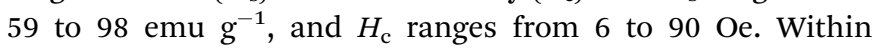
a single-phase magnetic material system, $H_{\mathrm{c}}$ can be tuned primarily through control of grain size, while magnetization can

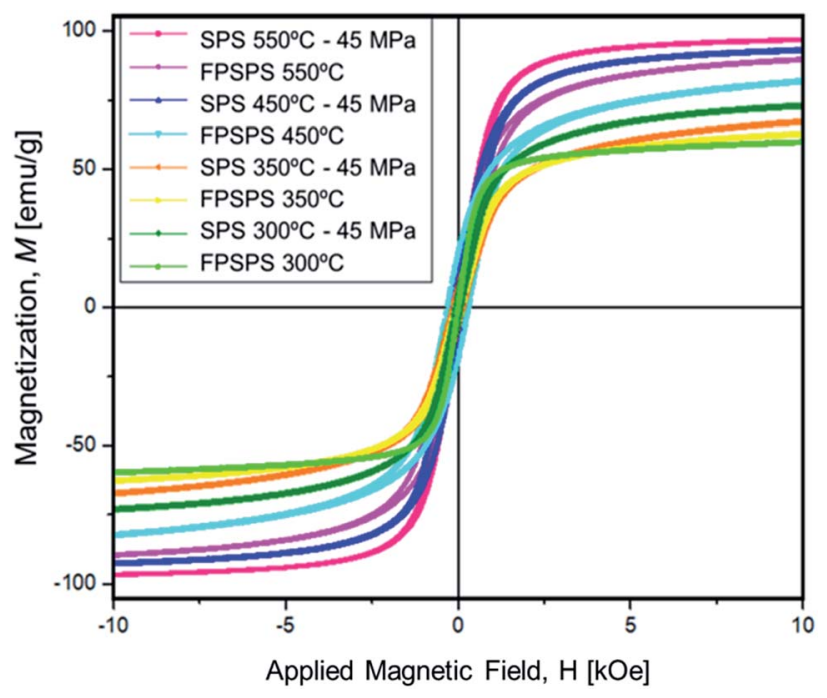

Fig. 7 VSM-obtained hysteresis magnetization/applied field curves for varying SPS parameters.

be tuned by varying volumetric concentration of the magnetic content, such as varying the ratio of organic content to iron oxide. Further tunability of $H_{\mathrm{c}}$ and $M_{\mathrm{s}}$ can be achieved by varying phase content as well, due to the diverse range of magnetic properties within the $\mathrm{Fe}-\mathrm{O}$ material system. In the following we focus on saturation magnetization, because of the high values obtained.

Saturation magnetization progressively increases when removing the oleic acid, which is diamagnetic, as Fig. 8 shows. This is a result of the increasing SPS temperatures applied during processing. The higher scatter characteristic of the $450{ }^{\circ} \mathrm{C}$ case is due to the higher number of samples processed at this temperature, with varying holding times, heating and cooling ramps. In the same plot, the values of saturation magnetization typical not only of magnetite $\left(\mathrm{Fe}_{3} \mathrm{O}_{4}\right)$, but of maghemite too $\left(\gamma-\mathrm{Fe}_{2} \mathrm{O}_{3}\right)$, are added as horizontal bands. It clearly emerges that the obtained pellets reach very high magnetization values, at the upper range of what previously reported for magnetite and maghemite, the ferrimagnetic $\mathrm{Fe}-\mathrm{O}$ phases. The oleic acid content is here plotted as wt $\%$ (value 


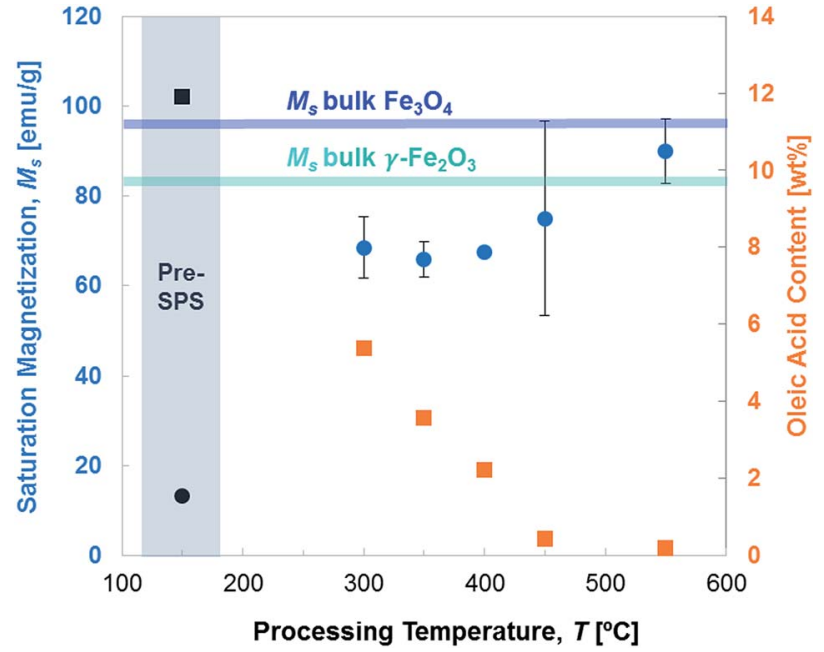

Fig. 8 Correlation between saturation magnetization, oleic acid content and processing temperature, compared with saturation magnetizations of bulk magnetite $\left(\mathrm{Fe}_{3} \mathrm{O}_{4}\right)$ and maghemite $\left(\gamma-\mathrm{Fe}_{2} \mathrm{O}_{3}\right)$. Square points correspond to oleic acid content, dots to saturation magnetization.

measured via TGA) instead of vol\% because of the units selected for magnetization $\left(\mathrm{emu} \mathrm{g}^{-1}\right)$.

Such an increase in saturation magnetization is, however, not only due to the removal of the diamagnetic organic phase, but also to a progressive transformation of $\gamma-\mathrm{Fe}_{2} \mathrm{O}_{3}$ into $\mathrm{Fe}_{3} \mathrm{O}_{4}$. The presence of $\gamma-\mathrm{Fe}_{2} \mathrm{O}_{3}$ is minor and not trivial to detect, since it shares the cubic atomic structure and the ferrimagnetic behavior of $\mathrm{Fe}_{3} \mathrm{O}_{4}$, making it difficult to observe both with X-ray scattering and through tell-tale kinks that characterize exchange coupling between different magnetic phases in the
VSM curves. ${ }^{27,63,64}$ The functionalized iron oxide nanoparticles are synthesized with the progressive oxidation of $\mathrm{FeO}$ into $\mathrm{Fe}_{3} \mathrm{O}_{4} \cdot{ }^{21}$ If the oxidation reaction keeps progressing, the formation of $\mathrm{Fe}_{2} \mathrm{O}_{3}$ can be expected.

Raman spectroscopy confirmed the initial presence of $\gamma$ $\mathrm{Fe}_{2} \mathrm{O}_{3}$ for all the employed material batches, as shown in Fig. 9A. ${ }^{65,66}$ Solid lines mark the peaks relative to $\mathrm{Fe}_{3} \mathrm{O}_{4}$, while dashed lines the ones relative to $\gamma-\mathrm{Fe}_{2} \mathrm{O}_{3}$. Magnetite is the dominant phase in all cases, but traces of maghemite appear in all three pre-SPS pellets (three lowest spectra in the figure), and are gradually smeared out when increasing the applied SPS temperature. The SPS environment is indeed a reducing atmosphere (vacuum conditions). The conversion of $\gamma-\mathrm{Fe}_{2} \mathrm{O}_{3}$ into $\mathrm{Fe}_{3} \mathrm{O}_{4}$ upon SPS processing is a remarkable result,, ${ }^{27,67}$ as it allows control of iron oxide phase content during the manufacturing of the material, and explains how above $450{ }^{\circ} \mathrm{C}$ the measured saturation magnetization can reach the maximum values typical of magnetite. Note that the presence of $\gamma-\mathrm{Fe}_{2} \mathrm{O}_{3}$ could also be detected with WAXS. Here, too, increasing processing temperatures correspond to the removal of this phase, see Fig. 9B. ${ }^{68,69}$ WAXS also shows $\mathrm{Fe}_{3} \mathrm{O}_{4}$ peaks' sharpening with increasing processing temperature, reflecting the grain growth of the iron oxide particles, in line with what observed via SEM (Fig. 4). Raman spectroscopy and WAXS therefore agree on confirming what previously observed via XPS, namely magnetite $\left(\mathrm{Fe}_{3} \mathrm{O}_{4}\right)$ being the dominant phase component of the ceramic nanoparticles, ${ }^{21}$ but also reveal the presence of a minor component of maghemite $\left(\gamma-\mathrm{Fe}_{2} \mathrm{O}_{3}\right)$, likely located on the surface of the nanoparticles, due to excessive oxidation during synthesis. Note that WAXS was also applied to investigate on the presence of metallic iron, which could be causing such high levels of saturation magnetization (see Fig. 7 and 8), but no trace was detected. This further confirms the
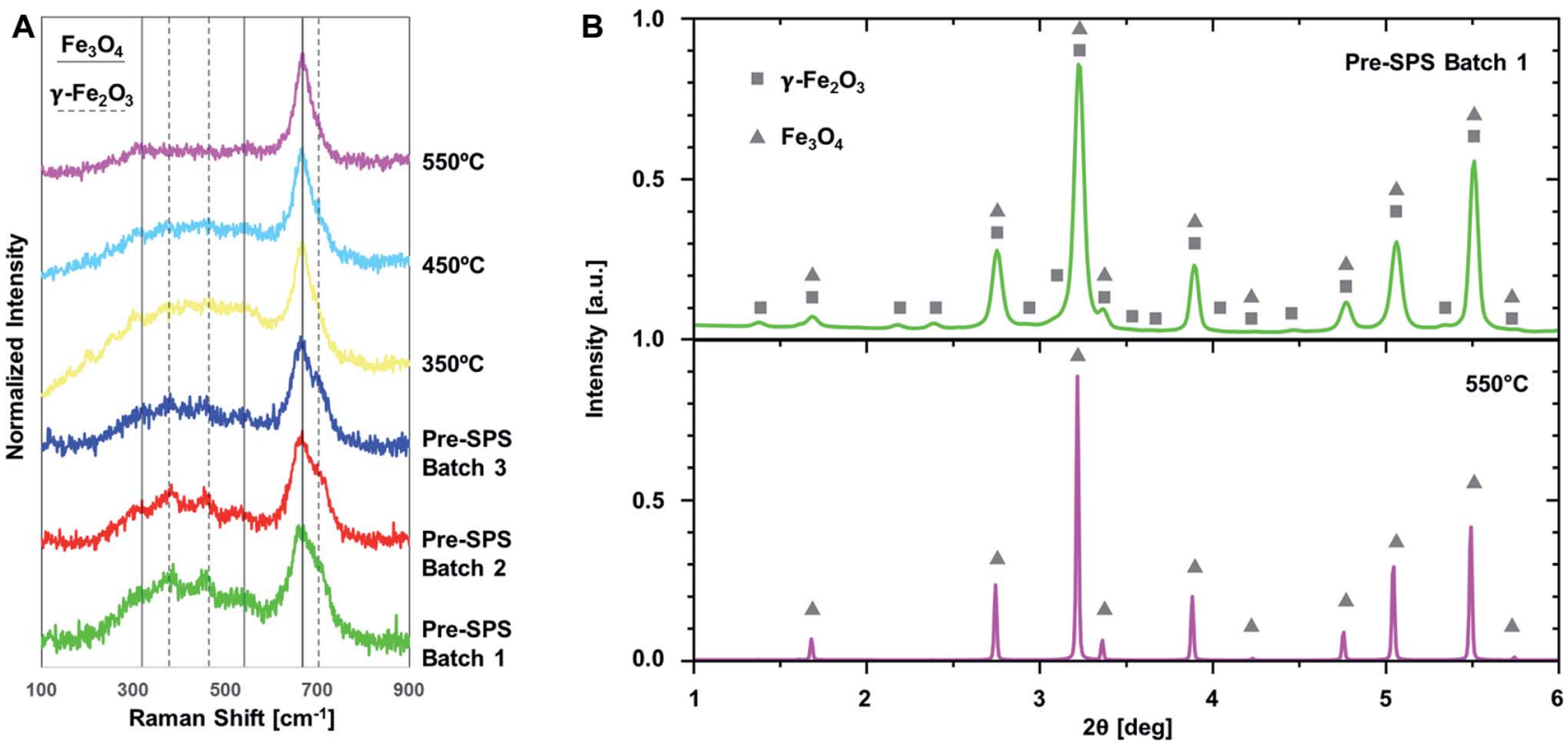

Fig. 9 Iron oxide phases present in the various samples as detected by Raman spectroscopy (A) and WAXS (B). A prevalent content of Fe $3 \mathrm{O}_{4}$, with some traces of $\gamma-\mathrm{Fe}_{2} \mathrm{O}_{3}$ that become gradually reduced at increasing temperatures, is observed. 
predominance of magnetite phase and the removal of $\gamma-\mathrm{Fe}_{2} \mathrm{O}_{3}$, having the latter a lower value of saturation magnetization with respect to $\mathrm{Fe}_{3} \mathrm{O}_{4}$.

Raman spectroscopy also revealed interesting details on the evolution of the organic phase during processing. At higher Raman frequencies, shown in Fig. 10, one can observe a drastic change between materials before and after SPS. The peaks highlighted with grey bands in the spectra correspond to amorphous carbon, and specifically to the so-called D and G bands (at 1350 and $1600 \mathrm{~cm}^{-1}$, respectively). The $\mathrm{G}$ band is relative to $\mathrm{sp}^{2}$ hybridized carbon and the $\mathrm{D}$ band is a defectactivated band in $\mathrm{sp}^{2}$ hybridized carbon materials. ${ }^{70}$ The less sharp peak at $1450 \mathrm{~cm}^{-1}$ is also typical of carbon materials. ${ }^{71,72}$ They are absent in the pre-SPS samples, and become increasingly sharper shifting from 350 to 450 and $550{ }^{\circ} \mathrm{C}$. This implies that the oleic acid undergoes multiple changes during SPS at increasing temperatures, including evaporation (at $\sim 360{ }^{\circ} \mathrm{C}$, depending on the local configuration and interactions among the ligand molecules) and decomposition, leading to the presence of a thin layer of amorphous carbon at the interfaces among iron oxide nanoparticles. Amorphous carbon is the result of the oleic acid decomposition at these high temperatures, and has very interesting implications, such as a potential significant increase in the material's thermal conductivity. This is an important parameter for the magnetic performance of the bulk iron oxides, since exposure to high temperatures is detrimental for the magnetic properties, and enhancing the capability of the material to conduct heat broadens their application spectrum. ${ }^{28}$ It is worth mentioning explicitly that Raman spectroscopy was performed on sample portions far from the pellets' surfaces, to exclude the effect of contamination from the graphite foil that surrounds the samples during SPS.

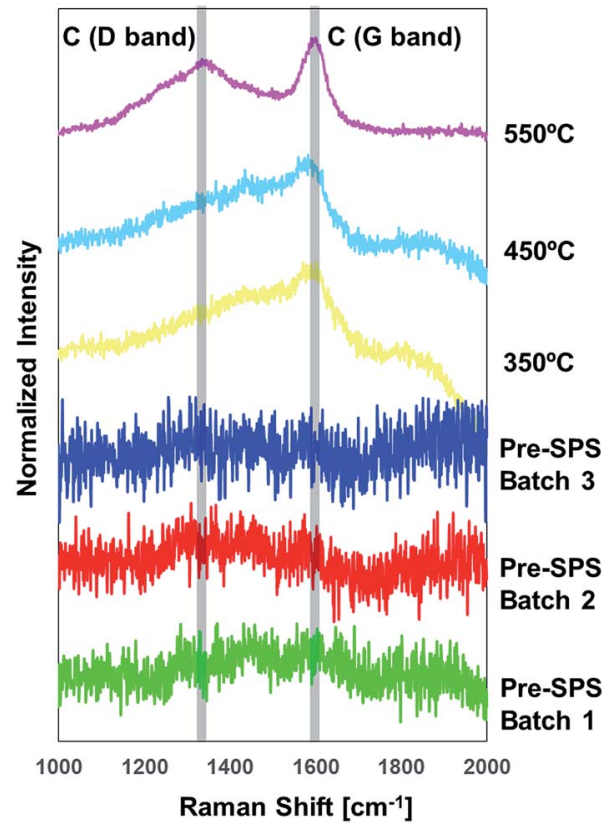

Fig. 10 Amorphous carbon traces in the various samples as detected by Raman spectroscopy.
Even more interestingly, the magnetic behavior of these bulk iron oxide-based materials can be further tuned to achieve superparamagnetism (SP), a feature typically restricted to nanopowders or nano-particulate materials. This is possible thanks to the nano-size of the iron oxide particles, for which the maximum size limit for superparamagnetism to be preserved is $20 \mathrm{~nm} .{ }^{73}$ For this purpose, a sample made with Batch 3 was selected, since it had the smallest average particle size $(15.7 \mathrm{~nm}$ diameter). In order to achieve consolidation in a bulk with good mechanical properties while preserving grain size below the 20 $\mathrm{nm}$-limit, the following SPS routine was applied: a heating ramp of $100{ }^{\circ} \mathrm{C} \mathrm{min}{ }^{-1}$ was used to first achieve a temperature of $250{ }^{\circ} \mathrm{C}$, held for 4 minutes in order to remove the oleic acid, and subsequently the maximum temperature of $450{ }^{\circ} \mathrm{C}$, a step immediately followed by accelerated cooling with argon flow. No pressure was applied. Note that this procedure ensures the removal of the organic phase, which is diamagnetic and thus dilutes the overall volumetric magnetization of the final material.

The resulting material's features are summarized in Fig. 11. Supercrystallinity was preserved, as the SEM micrograph shows. A very high saturation magnetization of $93 \mathrm{emu}^{-1}$ was achieved, while the coercivity was only $\sim 5 \mathrm{Oe}$, which is well within the range of coercivities typically reported for SP materials.

The obtained elastic modulus and hardness of $41.4 \mathrm{GPa}$ and $2.56 \mathrm{GPa}$, respectively, confirm the onset of necking. It is worth mentioning explicitly here that when the same SPS routine was applied to a sample of Batch 2, namely with larger particle size
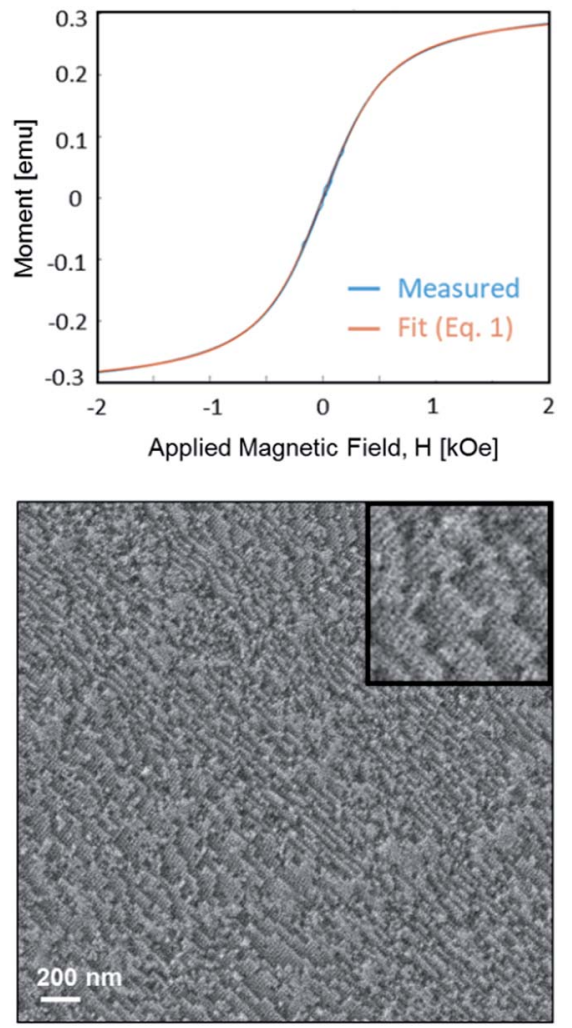

Fig. 11 Magnetization/applied field curve showing superparamagnetic behavior with the respective material's supercrystalline nanostructure. 
(18.4 $\mathrm{nm})$ and broader size scatter, a higher coercivity was obtained (11 Oe), corresponding to final particle sizes above $20 \mathrm{~nm}$.

If a sample behaves like a superparamagnet, its high temperature magnetization (at $T \gg T_{\mathrm{b}}$, blocking temperature) should be given by $M=n \mu \mathscr{L}(a),{ }^{74,75}$ where $n$ is the number of particles per unit volume, $\mu=M_{\mathrm{s}} V$ is the magnetic moment per particle, $\mathscr{L}$ is the Langevin function and $a=\mu H / k_{\mathrm{B}} T$. Thus, the magnetization for a superparamagnet can be written as

$$
M=n \mu\left[\operatorname{coth}\left(\frac{\mu H}{k_{\mathrm{B}} T}\right)-\frac{k_{\mathrm{B}} T}{\mu H}\right]
$$

Fig. 11 shows the material's measured $M-H$ curve and the results of a fit to eqn (1). The fit is excellent, confirming that this material with a retained supercrystalline structure is a bulk super-paramagnet.

To the best of the authors' knowledge, this is the first magnetite-based bulk superparamagnetic material produced, the largest previously reported reaching a maximum size of $174 \mathrm{~nm} .^{\mathbf{1 8 4 8}}$ The uniqueness of this material lies also in its excellent mechanical properties, implying material integrity, and in the fact that the partial necking between the nanoparticles is still not sufficient to allow the magnetic interactions responsible for the loss of superparamagnetism in bulk materials.

The advantage of superparamagnetism is a large magnetic response that is very easily switchable (reversible), as opposed to most magnetically responsive materials in which a coercive magnetic field must be surpassed before the magnetism is reversed. This has enabled numerous applications of SP materials in powder form including magnetic separation, drug delivery, etc. The existence of 'bulk' SP is exceedingly rare since superparamagnetism arises from size confinement observed only in nanomaterials. The materials developed here combine attributes of nanomagnets with the mechanical ease of use offered by bulk materials. In addition, since these large-scale structures are oxide based, they offer chemical and temperature stability. Potential applications are electrically insulating high magnetic response substrates and magnetic cores for transformers in energy applications. Such devices find, indeed, a major contributor to inefficiencies in hysteretic losses. Generally, a higher coercivity material will lead to greater energy losses due to the materials' inherent resistance to switching, which is often manifested in overheating of a transformer. In commercial transformers, $\mathrm{Fe}-\mathrm{Ni}$, $\mathrm{Fe}-\mathrm{Co}$, or other ferrite-based magnets are typically used, depending on the specific application. Fe-Ni and Fe-Co are materials of choice thanks to their high magnetic moment, high magnetic permeability and low coercivity. While a superparamagnetic material would fit these criteria perfectly, the difficulty has often been consolidating the material in such a way as to maximize the volumetric magnetization without undesired grain growth, reactions and phase changes. By preserving the superparamagnetic properties in a bulk magnet - as the processing routine presented here allows - it is possible to maximize the volumetric magnetization while retaining the low coercivity.

\section{Conclusions}

Bulk nanostructured iron oxide materials with an enhanced combination of magnetic and mechanical properties have been developed. The processing routine consists of a sequence of self-assembly of organically-functionalized iron oxide nanoparticles with narrow and monomodal size distribution, followed by spark plasma sintering (SPS). The self-assembly step leads to the formation of long-range order supercrystalline structures, and the fast heating achieved via SPS allows supercrystallinity preservation up to temperatures higher than those applicable with conventional furnace heating. The combination of organic material removal, partial densification and nanograin size retention can be tuned by varying the SPS parameters, to achieve high values of hardness and elastic modulus (approaching the values of bulk iron oxide even in porous samples) and of saturation magnetization. First-of-a-kind bulk superparamagnets were also obtained, preserving the supercrystalline structure with grain size below $20 \mathrm{~nm}$ to annihilate the material's coercivity, while maximizing saturation magnetization via organic removal and guaranteeing mechanical integrity via onset of necking phenomena.

\section{Contributions}

D. G. designed the study. D. G., B. B. and B. D. planned and performed the supercrystalline samples processing. D. G., E. T., K. T. C. and E. A. O. conducted and supervised the SPS tests. K. T. C., M. S. and J. E. G. conducted and supervised the magnetic characterization. D. G. and B. B. planned and performed the mechanical characterization. M. B. and M. M. performed and supervised the SAXS and WAXS analyses. L. S. performed the Raman spectroscopy. D. G. and G. A. S. supervised the study. All authors have contributed to discussion and paper preparation.

\section{Conflicts of interest}

There are no conflicts to declare.

\section{Acknowledgements}

The authors gratefully acknowledge financial support from the Deutsche Forschungsgemeinschaft (DFG, German Research Foundation) - Projektnummer 192346071 - SFB 986. D. G. gratefully acknowledges the support from the Alexander von Humboldt Foundation. The San Diego State University researchers gratefully acknowledge the support by the US Department of Energy, Materials Sciences Division, under Award No. DE-SC0008581. B. B. gratefully acknowledges the support from the Ministry of National Education of the Republic of Turkey. The authors are very thankful to Prof. Nadine Millot (Université Bourgogne Franche-Comté) and Dr. Kaline Furlan (Hamburg University of Technology) for the stimulating discussions and valuable input provided during the course of this study. 


\section{References}

1 E. Sturm née Rosseeva and H. Cölfen, Mesocrystals. Past, Present, Future, Crystals, 2017, 7, 207.

2 M. A. Boles, M. Engel and D. V. Talapin, Self-Assembly of Colloidal Nanocrystals, Chem. Rev., 2016, 116, 11220-11289.

3 T. Tachikawa and T. Majima, Metal oxide mesocrystals with tailored structures and properties for energy conversion and storage applications, NPG Asia Mater., 2014, 6, e100.

4 D. V. Talapin, J.-S. Lee, M. V. Kovalenko and E. V. Shevchenko, Prospects of Colloidal Nanocrystals for Electronic and Optoelectronic Applications, Chem. Rev., 2010, 110, 389-458.

5 A. S. Baimuratov, I. D. Rukhlenko, V. K. Turkov, A. V. Baranov and A. V. Fedorov, Quantum-dot supercrystals for future nanophotonics, Sci. Rep., 2013, 3, 1727.

6 J. Park, K. An, Y. Hwang, J.-G. Park, H.-J. Noh, J.-Y. Kim, J.-H. Park, N.-M. Hwang and T. Hyeon, Ultra-large-scale syntheses of monodisperse nanocrystals, Nat. Mater., 2004, 3, 891-895.

7 A. Feld, A. Weimer, A. Kornowski, N. Winckelmans, J.-P. Merkl, H. Kloust, R. Zierold, C. Schmidtke, T. Schotten, M. Riedner, S. Bals and H. Weller, Chemistry of Shape-Controlled Iron Oxide Nanocrystal Formation, ACS Nano, 2019, 13, 152-162.

8 J. Cheon, N.-J. Kang, S.-M. Lee, J.-H. Lee, J.-H. Yoon and S. J. Oh, Shape Evolution of Single-Crystalline Iron Oxide Nanocrystals, J. Am. Chem. Soc., 2004, 126, 1950-1951.

9 S. Laurent, D. Forge, M. Port, A. Roch, C. Robic, L. Vander Elst and R. N. Muller, Magnetic iron oxide nanoparticles, Chem. Rev., 2008, 108, 2064-2110.

10 M. D. Bentzon, J. van Wonterghem, S. Mørup, A. Thölén and C. J. W. Koch, Ordered aggregates of ultrafine iron oxide particles, Philos. Mag., 2007, 60, 169-178, DOI: 10.1080/ 13642818908211188.

11 J. Brunner, I. A. Baburin, S. Sturm, K. Kvashnina, A. Rossberg, T. Pietsch, S. Andreev, E. Sturm née Rosseeva and H. Cölfen, Self-Assembled Magnetite Mesocrystalline Films, Adv. Mater. Interfaces, 2017, 4, 1600431.

12 S. Disch, E. Wetterskog, R. P. Hermann, G. Salazar-Alvarez, P. Busch, T. Brückel, L. Bergström and S. Kamali, Shape Induced Symmetry in Self-Assembled Mesocrystals of Iron Oxide Nanocubes, Nano Lett., 2011, 11, 1651-1656.

13 Z. Fu, Y. Xiao, A. Feoktystov, V. Pipich, M.-S. Appavou, Y. Su, E. Feng, W. Jin and T. Brückel, Field-induced self-assembly of iron oxide nanoparticles investigated using small-angle neutron scattering, Nanoscale, 2016, 8, 18541-18550.

14 C. Jiang, S. M. Ng, C. W. Leung and P. W. T. Pong, Magnetically assembled iron oxide nanoparticle coatings and their integration with pseudo-spin-valve thin films, $J$. Mater. Chem. C, 2017, 5, 252-263.

15 G. Singh, H. Chan, A. Baskin, E. Gelman, N. Repnin, P. Kral and R. Klajn, Self-assembly of magnetite nanocubes into helical superstructures, Science, 2014, 345, 1149-1153.

16 D. Toulemon, Y. Liu, X. Cattoën, C. Leuvrey, S. Bégin-Colin and B. P. Pichon, Enhanced Collective Magnetic Properties in 2D Monolayers of Iron Oxide Nanoparticles Favored by Local Order and Local 1D Shape Anisotropy, Langmuir, 2016, 32, 1621-1628.

17 L.-M. Wang, O. Petracic, E. Kentzinger, U. Rücker, M. Schmitz, X.-K. Wei, M. Heggen and T. Brückel, Strain and electric-field control of magnetism in supercrystalline iron oxide nanoparticle- $\mathrm{BaTiO}_{3}$ composites, Nanoscale, 2017, 9, 12957-12962.

18 G. Wilbs, M. Smik, U. Rücker, O. Petracic and T. Brückel, Macroscopic nanoparticle assemblies, Mater. Today, 2017, 4, S146-S153.

19 H. Zeng, J. Li, J. P. Liu, Z. L. Wang and S. Sun, Exchangecoupled nanocomposite magnets by nanoparticle selfassembly, Nature, 2002, 420, 395-398.

20 G. Zoppellaro, J. Tuček, R. Herchel, K. Šafářová and R. Zbořil, $\mathrm{Fe}_{3} \mathrm{O}_{4}$ nanocrystals tune the magnetic regime of the $\mathrm{Fe} / \mathrm{Ni}$ molecular magnet, Inorg. Chem., 2013, 52, 8144-8150.

21 A. Dreyer, A. Feld, A. Kornowski, E. D. Yilmaz, H. Noei, A. Meyer, T. Krekeler, C. Jiao, A. Stierle, V. Abetz, H. Weller and G. A. Schneider, Organically linked iron oxide nanoparticle supercrystals with exceptional isotropic mechanical properties, Nat. Mater., 2016, 15, 522-528.

22 A. Navrotsky, L. Mazeina and J. Majzlan, Size-driven structural and thermodynamic complexity in iron oxides, Science, 2008, 319, 1635-1638.

23 R. M. Cornell and U. Schwertmann, The Iron Oxides, WileyVCH Verlag GmbH \& Co. KGaA, Weinheim, FRG, 2003.

24 S. Sakurai, A. Namai, K. Hashimoto and S.-I. Ohkoshi, First observation of phase transformation of all four $\mathrm{Fe}(2) \mathrm{O}(3)$ phases (gamma-epsilon-beta-alpha-phase), J. Am. Chem. Soc., 2009, 131, 18299-18303.

25 K. T. Chan, J. R. Morales, Y. Kodera and J. E. Garay, A processing route for bulk, high coercivity, rare-earth free, nanocomposite magnets based on metastable iron oxide, $J$. Mater. Chem. C, 2017, 5, 7911-7918.

26 J. R. Morales, J. E. Garay, M. Biasini and W. P. Beyermann, Magnetic characterization of bulk nanostructured iron oxides, Appl. Phys. Lett., 2008, 93, 22511.

27 J. R. Morales, S. Tanju, W. P. Beyermann and J. E. Garay, Exchange bias in large three dimensional iron oxide nanocomposites, Appl. Phys. Lett., 2010, 96, 13102.

28 S. Ramirez, K. Chan, R. Hernandez, E. Recinos, E. Hernandez, R. Salgado, A. G. Khitun, J. E. Garay and A. A. Balandin, Thermal and magnetic properties of nanostructured densified ferrimagnetic composites with graphene - graphite fillers, Mater. Des., 2017, 118, 75-80.

29 S. Behrens and I. Appel, Magnetic nanocomposites, Curr. Opin. Biotechnol., 2016, 39, 89-96.

30 P. Saravanan, J.-H. Hsu, D. Sivaprahasam and S. V. Kamat, Structural and magnetic properties of $\gamma-\mathrm{Fe}_{2} \mathrm{O}_{3}$ nanostructured compacts processed by spark plasma sintering, J. Magn. Magn. Mater., 2013, 346, 175-177.

31 C. Fei, Y. Zhang, Z. Yang, Y. Liu, R. Xiong, J. Shi and X. Ruan, Synthesis and magnetic properties of hard magnetic $\left(\mathrm{CoFe}_{2} \mathrm{O}_{4}\right)$-soft magnetic $\left(\mathrm{Fe}_{3} \mathrm{O}_{4}\right)$ nano-composite ceramics by SPS technology, J. Magn. Magn. Mater., 2011, 323, 18111816. 
32 E. K. Papynov, O. O. Shichalin, A. A. Belov, A. S. Portnyagin, V. Y. Mayorov, E. A. Gridasova, A. V. Golub, A. S. Nepomnyashii, I. G. Tananaev and V. A. Avramenko, Synthesis of nanostructured iron oxides and new magnetic ceramics using sol-gel and SPS techniques, AIP Conf. Proc., 2017, 1809, 20043.

33 E. A. Olevsky and D. V. Dudina, Field-Assisted Sintering: Science and Applications, Springer, Cham, Switzerland, 2018.

34 J. E. Alaniz, A. D. Dupuy, Y. Kodera and J. E. Garay, Effects of applied pressure on the densification rates in currentactivated pressure-assisted densification (CAPAD) of nanocrystalline materials, Scr. Mater., 2014, 92, 7-10.

35 J. E. Garay, Current-Activated, Pressure-Assisted Densification of Materials, Annu. Rev. Mater. Res., 2010, 40, 445-468.

36 J. R. Morales, N. Amos, S. Khizroev and J. E. Garay, Magnetooptical Faraday effect in nanocrystalline oxides, J. Appl. Phys., 2011, 109, 93110.

37 E. A. Olevsky, S. Kandukuri and L. Froyen, Consolidation enhancement in spark-plasma sintering, J. Appl. Phys., 2007, 102, 114913.

38 Z. A. Munir, D. V. Quach and M. Ohyanagi, Electric Current Activation of Sintering, J. Am. Ceram. Soc., 2011, 94, 1-19.

39 R. Orrù, R. Licheri, A. M. Locci, A. Cincotti and G. Cao, Consolidation/synthesis of materials by electric current activated/assisted sintering, Mater. Sci. Eng., R, 2009, 63, 127-287.

40 E. Olevsky, S. Kandukuri and L. Froyen, Analysis of Mechanisms of Spark-Plasma Sintering, Key Eng. Mater., 2008, 368-372, 1580-1584.

41 O. Guillon, J. Gonzalez-Julian, B. Dargatz, T. Kessel, G. Schierning, J. Räthel and M. Herrmann, Field-Assisted Sintering Technology/Spark Plasma Sintering, Adv. Eng. Mater., 2014, 16, 830-849.

42 D. Giuntini, R. K. Bordia and E. A. Olevsky, Feasibility of in situ de-agglomeration during powder consolidation, J. Am. Ceram. Soc., 2018, 443, 25.

43 D. Giuntini, E. A. Olevsky, C. Garcia-Cardona, A. L. Maximenko, M. S. Yurlova, C. D. Haines, D. G. Martin and D. Kapoor, Localized Overheating Phenomena and Optimization of Spark-Plasma Sintering Tooling Design, Materials, 2013, 6, 2612-2632.

44 A. E. Berkowitz, W. J. Schuele and P. J. Flanders, Influence of Crystallite Size on the Magnetic Properties of Acicular $\gamma$ $\mathrm{Fe}_{2} \mathrm{O}_{3}$ Particles, J. Appl. Phys., 1968, 39, 1261-1263.

45 M. I. Dar and S. A. Shivashankar, Single crystalline magnetite, maghemite, and hematite nanoparticles with rich coercivity, RSC Adv., 2014, 4, 4105-4113.

46 A. Teleki, M. Suter, P. R. Kidambi, O. Ergeneman, F. Krumeich, B. J. Nelson and S. E. Pratsinis, Hermetically Coated Superparamagnetic $\mathrm{Fe}_{2} \mathrm{O}_{3}$ Particles with $\mathrm{SiO}_{2}$ Nanofilms, Chem. Mater., 2009, 21, 2094-2100.

47 J. M. D. Coey and D. Khalafalla, Superparamagnetic $\gamma-\mathrm{Fe}_{2} \mathrm{O}_{3}$, Phys. Status Solidi A, 1972, 11, 229-241.

48 J. Ge, Y. Hu, M. Biasini, W. P. Beyermann and Y. Yin, Superparamagnetic magnetite colloidal nanocrystal clusters, Angew. Chem., 2007, 46, 4342-4345.
49 S.-J. Lee, J.-R. Jeong, S.-C. Shin, J.-C. Kim and J.-D. Kim, Synthesis and characterization of superparamagnetic maghemite nanoparticles prepared by coprecipitation technique, J. Magn. Magn. Mater., 2004, 282, 147-150.

50 R. Zboril, M. Mashlan and D. Petridis, Iron(III) Oxides from Thermal Processes. Synthesis, Structural and Magnetic Properties, Mössbauer Spectroscopy Characterization, and Applications, Chem. Mater., 2002, 14, 969-982.

51 M. Mikhaylova, D. K. Kim, N. Bobrysheva, M. Osmolowsky, V. Semenov, T. Tsakalakos and M. Muhammed, Superparamagnetism of Magnetite Nanoparticles, Langmuir, 2004, 20, 2472-2477.

52 N. Schell, A. King, F. Beckmann, T. Fischer, M. Müller and A. Schreyer, The High Energy Materials Science Beamline (HEMS) at PETRA III, Mater. Sci. Forum, 2013, 772, 57-61.

53 A. P. Hammersley, FIT2D: An Introduction and Overview. Internal Report ESRF97HA02T, ESRF, Grenoble, France, 1997.

54 S. Förster, L. Apostol and W. Bras, Scatter, J. Appl. Crystallogr., 2010, 43, 639-646.

55 L. Lutterotti, Total pattern fitting for the combined sizestrain-stress-texture determination in thin film diffraction, Nucl. Instrum. Methods Phys. Res., Sect. B, 2010, 268, 334-340.

56 Y. Q. Fu, C. Shearwood, B. Xu, L. G. Yu and K. A. Khor, Characterization of spark plasma sintered $\mathrm{Ag}$ nanopowders, Nanotechnology, 2010, 21, 115707.

57 S.-Y. Zhao, D. K. Lee, C. W. Kim, H. G. Cha, Y. H. Kim and Y. S. Kang, Synthesis of Magnetic Nanoparticles of $\mathrm{Fe}_{3} \mathrm{O}_{4}$ and $\mathrm{CoFe}_{2} \mathrm{O}_{4}$ and Their Surface Modification by Surfactant Adsorption, Bull. Korean Chem. Soc., 2006, 27, 237-242.

58 B. Domènech, M. Kampferbeck, E. Larsson, T. Krekeler, B. Bor, D. Giuntini, M. Blankenburg, M. Ritter, M. Müller, T. Vossmeyer, H. Weller and G. A. Schneider, Hierarchical supercrystalline nanocomposites through the self-assembly of organically-modified ceramic nanoparticles, Sci. Rep., 2019, 9, 1516.

59 Y. Dong and I.-W. Chen, Grain Growth with Size-Dependent or Statistically Distributed Mobility, 2017, available at: arXiv preprint arXiv: 1708.04092 .

60 T. H. de Keijser, J. I. Langford, E. J. Mittemeijer and A. B. P. Vogels, Use of the Voigt function in a single-line method for the analysis of X-ray diffraction line broadening, J. Appl. Crystallogr., 1982, 15, 308-314.

61 D. Chicot, J. Mendoza, A. Zaoui, G. Louis, V. Lepingle, F. Roudet and J. Lesage, Mechanical properties of magnetite $\left(\mathrm{Fe}_{3} \mathrm{O}_{4}\right)$, hematite $\left(\alpha-\mathrm{Fe}_{2} \mathrm{O}_{3}\right)$ and goethite $(\alpha$ $\mathrm{FeO} \cdot \mathrm{OH})$ by instrumented indentation and molecular dynamics analysis, Mater. Chem. Phys., 2011, 129, 862-870.

62 G. M. Pharr, E. G. Herbert and Y. Gao, The Indentation Size Effect, Annu. Rev. Mater. Res., 2010, 40, 271-292.

63 E. E. Fullerton, J. Jiang and S. Bader, Hard/soft magnetic heterostructures, J. Magn. Magn. Mater., 1999, 200, 392-404.

64 J. Nogués, J. Sort, V. Langlais, V. Skumryev, S. Suriñach, J. S. Muñoz and M. D. Baró, Exchange bias in nanostructures, Phys. Rep., 2005, 422, 65-117.

65 I. Chamritski and G. Burns, Infrared- and Raman-active phonons of magnetite, maghemite, and hematite, J. Phys. Chem. B, 2005, 109, 4965-4968. 
66 S. P. Schwaminger, D. Bauer, P. Fraga-García, F. E. Wagner and S. Berensmeier, Oxidation of magnetite nanoparticles, CrystEngComm, 2017, 19, 246-255.

67 K. Witte, W. Bodnar, N. Schell, G. Fulda and E. Burkel, Phase transformations of stoichiometric mixtures of hematite and iron under FAST conditions, J. Alloys Compd., 2017, 724, 728734.

68 W. H. Bragg, The Structure of Magnetite and the Spinels, Nature, 1915, 95, 561.

69 E. Solano, C. Frontera, T. Puig, X. Obradors, S. Ricart and J. Ros, Neutron and X-ray diffraction study of ferrite nanocrystals obtained by microwave-assisted growth. A structural comparison with the thermal synthetic route, $J$. Appl. Crystallogr., 2014, 47, 414-420.

70 M. S. Dresselhaus, A. Jorio, A. G. Souza Filho and R. Saito, Defect characterization in graphene and carbon nanotubes using Raman spectroscopy, Philos. Trans. R. Soc., A, 2010, 368, 5355-5377.

71 A. C. Ferrari and J. Robertson, Raman spectroscopy of amorphous, nanostructured, diamond-like carbon, and nanodiamond, Philos. Trans. R. Soc., A, 2004, 362, 24772512.

72 V. Vorlíček, J. Rosa, M. Vaněček, M. Nesládek and L. M. Stals, Quantitative study of Raman scattering and defect optical absorption in CVD diamond films, Diamond Relat. Mater., 1997, 6, 704-707.

73 Y. Hou, J. Yu and S. Gao, Solvothermal reduction synthesis and characterization of superparamagnetic magnetite nanoparticles, J. Mater. Chem., 2003, 13, 1983.

74 C. P. Bean and J. D. Livingston, Superparamagnetism, J. Appl. Phys., 1959, 30, S120-S129.

75 B. D. Cullity and C. D. Graham, Introduction to magnetic materials, Wiley, Hoboken, N.J., Chichester, $2^{\text {nd }}$ ed., 2009. 